\title{
Investigating the structure of biomass-derived non-graphitising mesoporous carbons by electron energy loss spectroscopy in the transmission electron microscope and X-ray photoelectron spectroscopy
}

\author{
A.S. Marriott ${ }^{1,2,3}$, A.J. Hunt ${ }^{1,2}$, E. Bergström ${ }_{5 *}^{1,3}$, K. Wilson ${ }^{4}$, V.L. Budarin ${ }^{1,2}$, J. \\ Thomas-Oates $^{1,3}$, J. H. Clark ${ }^{1,2}$, R. Brydson ${ }^{5}$ \\ ${ }^{1}$ Department of Chemistry, ${ }^{2}$ Green Chemistry Centre of Excellence, ${ }^{3}$ Centre of Excellence in \\ Mass Spectrometry, University of York, York YO10 5DD, UK, \\ ${ }_{5}^{4}$ School of Chemistry, Cardiff University, Cardiff, UK, \\ ${ }^{5}$ Institute for Materials Research, School of Process, Environmental and Materials \\ Engineering (SPEME), University of Leeds, Leeds LS2 9JT, UK
}

\begin{abstract}
We have investigated the microstructure and bonding of two biomass-based porous carbon chromatographic stationary phase materials (alginic acid-derived Starbon ${ }^{\circledR}$ and calcium alginate-derived mesoporous carbon spheres (AMCS)) and a commercial porous graphitic carbon (PGC), using high resolution transmission electron microscopy, electron energy loss spectroscopy (EELS), $\mathrm{N}_{2}$ porosimetry and X-ray photoelectron spectroscopy (XPS). The planar carbon $s p^{2}$-content of all three material types is similar to that of traditional nongraphitising carbon although, both biomass-based carbon types contain a greater percentage of fullerene character (i.e curved graphene sheets) than a non-graphitising carbon pyrolysed at the same temperature. This is thought to arise during the pyrolytic breakdown of hexauronic acid residues into C5 intermediates. Energy dispersive X-ray and XPS analysis reveals a homogeneous distribution of calcium in the AMCS and a calcium catalysis mechanism is discussed. That both Starbon $^{\circledR}$ and AMCS, with high-fullerene character, show chromatographic properties similar to those of a commercial PGC material with extended graphitic stacks, suggests that, for separations at the molecular level, curved fullerene-like and planar graphitic sheets are equivalent in PGC chromatography. In addition, variation in the
\end{abstract}

* Corresponding author. Tel:+44(0)113 3432369. Email: r.m.drummondbrydson@leeds.ac.uk 
number of graphitic layers suggests that stack depth has minimal effect on the retention mechanism in PGC chromatography.

\section{Introduction}

Porous graphitic carbons (PGC) are attracting increasing interest for applications such as catalysis [1] and [2], adsorption [3], and energy storage [4] and [5]. In particular, PGC has attracted significant interest over the past decades as a liquid chromatography (LC) stationary phase, owing to its unique mechanism of separation of polar compounds under mass spectrometry-compatible reversed-phase conditions, as well as its capacity to function over the entire $\mathrm{pH}$ range[3], [6], [7] and [8]. PGC compares favourably with the widely adopted standard reversed-phase, chemically bonded but less stable silica columns, which are unable to retain polar compounds.

Over the last few years, the preparation of mesoporous carbonaceous material via thermal decomposition of expanded starch (which has been commercialised as Starbon ${ }^{\circledR}$ ) has been developed [9] and [10]. A second generation Starbon ${ }^{\circledR}$ prepared from the algal polysaccharide alginic acid (termed Starbon ${ }^{\circledR}$ AXXX, where "XXX" refers to the temperature of pyrolysis) has been shown to be effective in the LC separation of low mass carbohydrates [11], with retention comparable to that on a commercial PGC column [12]. Synthesis of alginic acidderived Starbon ${ }^{\circledR}$ involves the expansion of an alginic acid hydrogel in water, followed by solvent exchange with ethanol and supercritical $\mathrm{CO}_{2}$ drying to produce an aerogel precursor, which is then subjected to pyrolysis at up to $1000{ }^{\circ} \mathrm{C}$ under an inert atmosphere. Although attempts have been made to understand the carbon species present in these materials, the characterisation techniques used have been of an indirect nature, enabling only the description that the materials show extended aromaticity. Thus there is a need to adopt methods which allow the direct comparison of the carbon character within a commercial PGC material with 
that in an alginic acid-derived Starbon ${ }^{\circledR}$-material, in order to understand why alginic acidderived Starbon ${ }^{\circledR}$ shows retention similar to that on PGC. The purpose of this paper is therefore to shed light on the microstructure and chemical composition of alginic acid-derived Starbon $^{\circledR}$, applying transmission electron microscopy (TEM) imaging and diffraction techniques combined with compositional analysis using energy-dispersive X-ray (EDX) and electron energy loss (EELS) spectroscopy together with a semi-quantitative analysis of the EELS carbon K-edge, for the extraction of both the fraction of $s p^{2}$-bonded carbon atoms and the degree of fullerene character [13]. This data has been directly compared with similar data for a commercial PGC material. Two alginic acid-derived Starbon ${ }^{\circledR}$ samples were studied: one had been pyrolysed to $1000{ }^{\circ} \mathrm{C}$ (Starbon $\left.{ }^{\circledR} \mathrm{A} 1000\right)$ and the other to $800{ }^{\circ} \mathrm{C}$ (Starbon ${ }^{\circledR} \mathrm{A} 800$ ). These samples represent respectively materials prepared using the same pyrolysis temperature as White et al. [11] and the pyrolysis temperature more recently favoured for the preparation of Starbon ${ }^{\circledR}$ material [14].

Coupled to this, recent work aimed at improving the morphology of alginic acid-derived Starbon ${ }^{\circledR}$ particles for the purposes of packing chromatographic columns has led to the development of calcium alginate mesoporous carbon microspheres (AMCS), which are also pyrolysed to $800{ }^{\circ} \mathrm{C}$ under an inert atmosphere [15]. This research has highlighted the effect that calcium ions play in the structural properties of AMCS, where microporosity of the material was observed to increase as calcium levels decreased. To date, no analysis of the effect of calcium removal on the microstructure of these materials has been attempted. This paper therefore also attempts to analyse the nanostructure and chemical composition of two different AMCS samples; a unwashed sample (AMCS-NW; the sample has not undergone an acid-wash step to remove bound calcium ions), and a washed sample (AMCS-W; the sample was acid-washed using $0.1 \mathrm{M} \mathrm{HCl}$ ). The purpose was to draw a comparison between AMCS 
and the alginic acid-derived Starbon ${ }^{\circledR}$ materials whose morphology they have been designed to improve upon, as well as to study the effect that elevated calcium levels may have on the carbonisation of AMCS during pyrolysis.

\section{Experimental}

\subsection{Chemicals}

Alginic acid from brown algae and sodium alginate were purchased from Sigma (Poole, UK). Commercial PGC was purchased from Hypersil (Runcorn, UK). Calcium chloride and all solvents were purchased from Fisher (Fisher Scientific, Loughborough, UK).

\subsection{Alginic acid-derived Starbon ${ }^{\circledR}$ precursor synthesis}

Alginic acid from brown algae (Sigma) was gelatinised in distilled water: a suspension of the polysaccharide $\left(1 \mathrm{~g}\right.$ in $20 \mathrm{~mL}$ water) was stirred for two hours at $90{ }^{\circ} \mathrm{C}$. Retrogradation (whereby the polysaccharide chains realign themselves) by storing the solution at $5{ }^{\circ} \mathrm{C}$ for 24 $\mathrm{h}$ was performed in order to strengthen the resulting hydrogel. The water was exchanged for ethanol and the resulting alcogel dried in supercritical $\mathrm{CO}_{2}\left(\mathrm{scCO}_{2}\right)$. Drying was conducted using a Thar SFE-500 supercritical extractor heated to $40{ }^{\circ} \mathrm{C}$ and held at a pressure of 120 Bar for $2 \mathrm{~h}$ under dynamic flow conditions $\left(40 \mathrm{~g} \mathrm{~min}^{-1} \mathrm{CO}_{2}\right)$. The extractor was subsequently allowed to depressurise over a period of $12 \mathrm{~h}$.

\subsection{Preparation of AMCS precursor}

Sodium alginate (Sigma-Aldrich) was mixed in deionised water (1 $\mathrm{g}$ in $50 \mathrm{~mL}$ water) to produce a viscous solution. The solution was passed through an electrospray ionization emitter source that had been detached from the mass spectrometer, at a flow rate of $1 \mathrm{~mL} / \mathrm{min}$ using a constametric 3200 solvent delivery system (LDC Analytical). The solution was nebulized using 9 psi air, sprayed into a constantly-stirred $0.24 \mathrm{M}$ solution calcium chloride 
and cured in solution for $24 \mathrm{~h}$ to prepare hydrogel spheres of calcium alginate. The hydrogel spheres were transferred into deionised water and washed. At this stage, the AMCS-W sample spheres were acid-washed using hydrochloric acid $(0.1 \mathrm{M}$ or $0.5 \mathrm{M})$, as required, to reduce the calcium content of the material. The removal of water was performed by solvent exchange with ethanol (a lower surface tension solvent) using a method reported previously (Robitzer et al.) [16]. The excess solvent was removed from the resulting alcogel by Buchner filtration before the material was dried by $\mathrm{scCO}_{2}$. The drying was performed using a Thar SFE-500 supercritical extractor heated to $40{ }^{\circ} \mathrm{C}$ at a pressure of 120 Bar for $4 \mathrm{~h}$ under dynamic flow conditions (40 $\mathrm{g} \mathrm{min}^{-1} \mathrm{CO}_{2}$ ). The extractor was depressurized slowly over $12 \mathrm{~h}$.

\subsection{Preparation of carbonaceous alginic acid-derived Starbon ${ }^{\circledR} / A M C S$ material}

Starbon $^{\circledR}$ and AMCS precursors were pyrolysed by heating under a $\mathrm{N}_{2}$ atmosphere $\left(\mathrm{N}_{2}\right.$ flow:

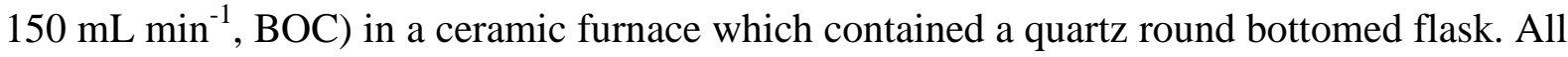
samples were pyrolysed to either $800{ }^{\circ} \mathrm{C}$ (Starbon ${ }^{\circledR}$ A800, AMCS-NW and AMCS-W) or $1000{ }^{\circ} \mathrm{C}\left(\right.$ Starbon $\left.^{\circledR} \mathrm{A} 1000\right)$ at a rate of $1 \mathrm{~K} \mathrm{~min}^{-1}$ followed by a hold of $20 \mathrm{~min}$.

\section{5. $N_{2}$ sorption porosimetry}

$\mathrm{N}_{2}$ porosimetry was performed on a Micromeritics Tristar volumetric apparatus at $77 \mathrm{~K}$. The samples were outgassed at $160{ }^{\circ} \mathrm{C}$ for $8 \mathrm{~h}$ under atmospheric pressure before analysis.

\subsection{Transmission electron microscopy (TEM)}

TEM characterisation was carried out with a FEI Supertwin CM200 thermally assisted field emission gun transmission electron microscope operated at $197 \mathrm{keV}$ (point resolution 0.24 $\mathrm{nm}$ ) equipped with an ultra thin window Oxford Instruments energy dispersive X-ray (EDX) detector running ISIS software and Gatan (GIF200) EELS imaging filter. TEM samples were 
prepared by grinding and ultrasonically dispersing the powders in methanol, one drop of the dispersion was deposited onto a copper grid (400 mesh) covered by a holey carbon-coated film (Agar Scientific), and the sample was dried in air for about 15-20 minutes. All EELS spectra were collected in diffraction mode from areas some $180 \mathrm{~nm}$ in diameter; core loss spectra were all collected using 'magic' collection angle conditions for which spectra are crystallographically orientation independent [17]. Carbon K-edge spectra were processed and fitted using the Digital Micrograph software package (Gatan). Fitting and analysis of the EELS carbon K-edge was performed using the approach described by Zhang et al. [13].

\subsection{X-ray photoelectron spectroscopy}

X-ray photoelectron spectroscopy (XPS) were performed on a Kratos AXIS HSi X-ray photoelectron spectrometer equipped with a monochromatic $\mathrm{Mg} \mathrm{K} \alpha \mathrm{X}$-ray source (energy $1253.6 \mathrm{eV}$ ) and a charge neutralisation gun. Spectra were recorded at an analyzer pass energy of $20 \mathrm{eV}$. Spectra were energy referenced to the C $1 s$ photoelectron peak at $284.6 \mathrm{eV}$, with quantification performed using CASA-XPS 2.3.15 following Shirley background subtraction. Sensitivity factors for C $1 s(0.318), \mathrm{Ca} 2 p(1.95), \mathrm{N} 1 s(0.505)$ and $\mathrm{O} 1 s(0.736)$ were used to correct the signal intensities and quantify the surface composition. Peak fitting for $\mathrm{Ca}$ and carbohydrate features was performed using a 30:70 Gaussian-Lorentzian peak shape, whilst deconvolution of graphitic-like sample data required an asymmetric Doniach Sunjic line shape. Spectra were fitted with the minimum number of components required to provide a good envelope, with full width half maxima (FWHM) kept fixed within each class of material. Typical FWHM values were $1.5-1.7 \mathrm{eV}$ for $\mathrm{Ca} 2 p$; while for the C $1 s$, FWHM were $2.5 \mathrm{eV}$ for the AMCS and Starbon ${ }^{\circledR}$ precursors and $1.5 \mathrm{eV}$ for the pyrolysed products. Binding energies for components were chosen using reference data from the NIST XPS database [18].

\section{Results and discussion}




\section{1: $N_{2}$ sorption porosimetry}

Table 1 summarises the surface area and pore structure characteristics of the samples as determined by $\mathrm{N}_{2}$ sorption porosimetry. All samples are predominantly mesoporous, with the commercial PGC material having the highest mesopore content at $94 \%$. Pore diameter is well within the mesopore range for all samples, although the commercial PGC material had a narrower pore size distribution (not shown). This is to be expected, as this material is synthesised using a hard-templating route, for which a template of a specified pore size is used [3].

\begin{tabular}{|c|c|c|c|c|}
\hline Sample & $\begin{array}{l}{ }^{a} \mathbf{S A}_{\mathrm{BET}} \\
/ \mathbf{m}^{2} \mathbf{g}^{-1}\end{array}$ & $\begin{array}{l}{ }^{b} \mathbf{P V} \text { Des } \\
/ \mathrm{cm}^{3} \mathbf{g}^{-1}\end{array}$ & $\begin{array}{l}{ }^{c} \text { PD } \\
/ \mathbf{n m}\end{array}$ & $\begin{array}{l}{ }^{d} \text { Meso. } \\
/ \%\end{array}$ \\
\hline Commercial PGC & 101 & 0.67 & 18.35 & 94 \\
\hline Starbon $^{\circledR}$ A800 & 396 & 1.78 & 21.04 & 91 \\
\hline Starbon $^{\circledR} \mathbf{A} 1000$ & 605 & 0.88 & 13.09 & 72 \\
\hline AMCS-NW & 562 & 1.20 & 12.49 & 81 \\
\hline AMCS-W & 515 & 2.23 & 19.80 & 90 \\
\hline \multicolumn{5}{|c|}{$\begin{array}{l}a=\mathrm{BET} \text { surface area; } b=\mathrm{BJH} \text { desorption pore volume; } c=\mathrm{BJH} \text { desorption pore diameter; } d=\text { Percentage } \\
\text { mesoporosity }\end{array}$} \\
\hline
\end{tabular}

Table 1: $\mathrm{N}_{2}$ sorption data for commercial PGC, AMCS and Starbon ${ }^{\circledR}$ samples

The alginic acid-based samples have greater surface area than the commercial PGC material as well as larger pore volumes, although there is significant variation among these samples. The porosity of the alginic acid-based carbons is derived from the porous expanded polysaccharide framework created during the synthesis gelling process, a so-called "naturaltemplate". Although not as strong as a hard-template (e.g. a silica bead), the synthesis has been developed to retain this framework throughout the synthesis without causing extensive structural collapse. However, the porous polysaccharide framework is susceptible to heat treatment which gives rise to micropore formation and the surface area values observed. 


\section{2: Transmission electron microscopy (TEM)}

\subsection{1: High resolution TEM}

Figure 1 shows high resolution transmission electron micrographs of: commercial PGC (Figure 1(a) and (b)); Starbon ${ }^{\circledR}$ A800 (Figure 1(c) and (d)); Starbon ${ }^{\circledR}$ A1000 (Figure 1(e) and (f)); AMCS-NW (Figure1(g) and (h)) and AMCS-W (Figure 1(i) and (j)). These images are representative of the microstructures observed within these materials at both low and high magnifications.

Commercial PGC exhibits a high proportion of disordered, closed loop structures of graphitic layer stacks exhibiting both rounded (i.e smooth) curvature and also very angular curvature (Figure 1 (a) and (b)). The graphitic stacks (consisting of (002) planes) are ca. 1-2 nm thick (3 to 6 graphene layers) and display a range of widths varying between 5 and15 $\mathrm{nm}$. Low magnification micrographs (Figure 1(a)) show the enclosed pore structure but also reveal that no long-range order graphitization exists at the macroscopic scale. These images are consistent with those reported by Zhang et al. [13] for non-graphitising carbons pyrolysed above $2000{ }^{\circ} \mathrm{C}$. This agrees also with the literature describing PGC synthesis, in which phenolic carbonising resins are pyrolysed at temperatures in excess of $2000{ }^{\circ} \mathrm{C}$ in order to reduce the micropore content [3]. Interestingly, there is also evidence of smaller, quasi-flat but highly-aligned carbon layers which appear similar to those described by Harris et al. for low temperature-pyrolysed graphitizing microporous carbons, derived from anthracene [19].

In the Starbon ${ }^{\circledR}$ A800 sample, there is only minimal ordering of (002) graphitic planes, limited to 2-3 aligned layers at most (Figure 1(c)). A high degree of rounded layer curvature is also evident, which indicates a large degree of fullerene-like character (i.e. the presence of carbon pentagons and heptagons in addition to normal graphitic hexagons) which results in a very 
closed-loop structure. At low magnification the material appears particulate in nature and the images show the porous, irregular-shaped nature of the alginic acid-derived Starbon ${ }^{\circledR}$ materials.

For Starbon ${ }^{\circledR}$ A1000, the regions sampled at low magnification (Figure 1(e)) display the same particulate microstructure and pore structure as observed for Starbon ${ }^{\circledR}$ A800. However, at higher magnification (Figure 1(f)), the fullerene-like character is more obvious with more distinct loop structures apparent, which exhibit slightly thicker stacks (between 3-5 graphene layers). Based on the observations at high magnification of short range graphitic order and limited graphite stacking, Starbon ${ }^{\circledR}$ A1000 appears to closely resemble a non-graphitising carbon pyrolysed to $1000{ }^{\circ} \mathrm{C}$ as described in the paper of Zhang et al. [13].

With regards to the AMCS samples, the carbon microstructures (Figures 1(h) and 1(j)) show extensive fullerene-like character with a high degree of smooth curvature, which is also found to enclose a more open network of pores. There is evidence for the presence of closed-loop structures in both samples, although the washed sample revealed slightly thicker layer packets (4-5 graphene layers), which were less obvious in the AMCS-NW material. However, it is at low magnification where the differences are clearer between the AMCS samples. Both samples differ from alginic acid-derived Starbon $^{\circledR}$ in that the structure is less an agglomeration of particulates and appears to be a more expanded carbon-based material; in particular, Figure 1(g) gives evidence for an extended open mesopore structure. Both AMCS materials show a high degree of mesoporosity, together with distinct, dense nanoparticulate regions (observed as dark areas, due to mass-thickness contrast in TEM bright field images); for AMCS-W, these nanoparticulate regions are homogeneously distributed throughout the material, whereas for AMCS-NW this homogeneous distribution is interspersed with additional larger crystalline nanoparticulate regions (as indicated by selected area electron 
diffraction, and which appear very dark in TEM bright field images due to diffraction contrast). EDX spectroscopic analysis of these samples in the TEM indicates a higher percentage of calcium in these regions of high density than in the more open (less dense) regions, suggesting that these nanoparticles are predominantly a calcium species. The chemical nature of these nanoparticles and crystals were investigated by XPS and are discussed below (see Section 3.3). 


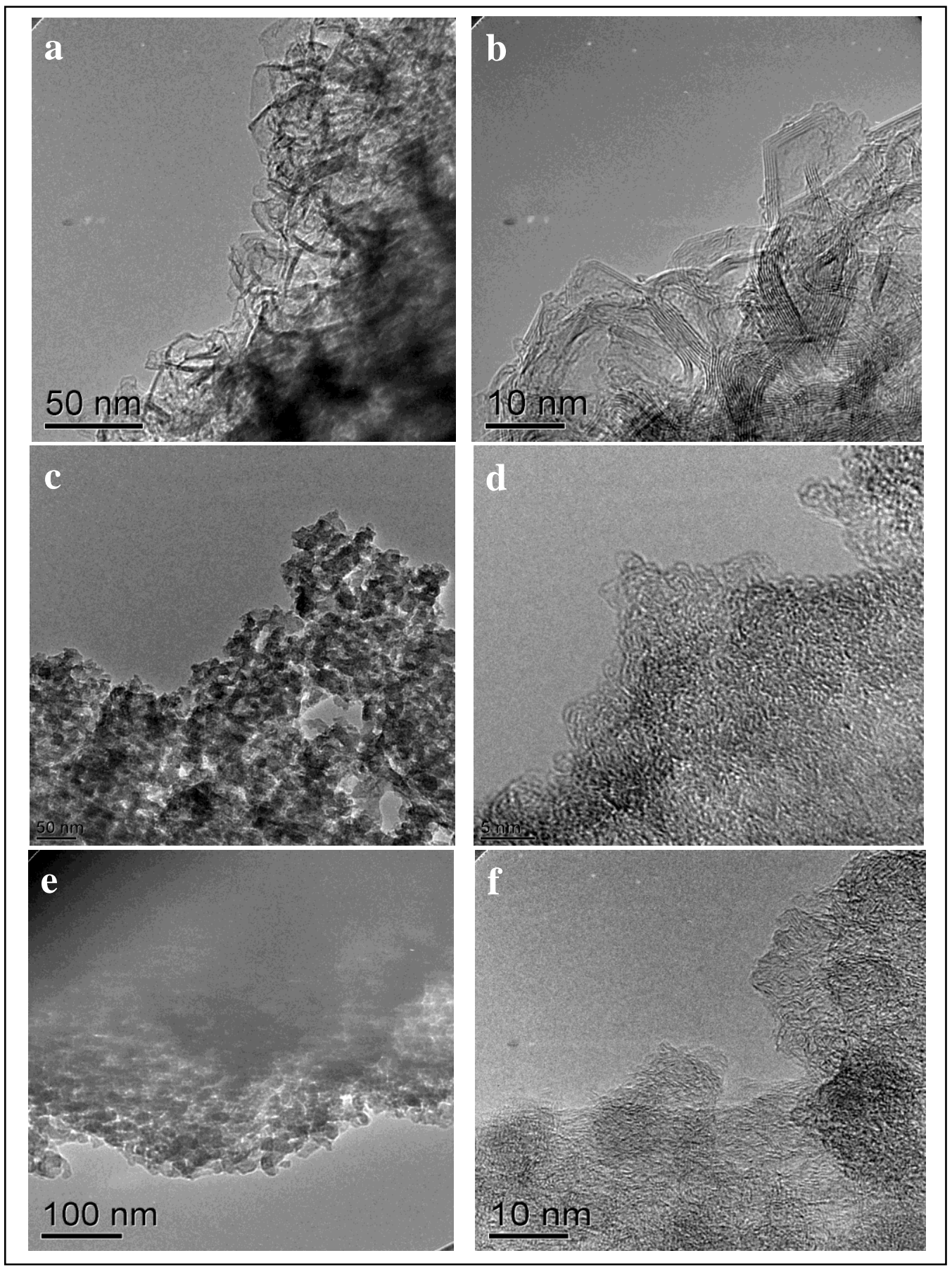

Figure 1: High resolution TEM images of commercial PGC at a) low and b) high magnification; Starbon ${ }^{\circledR}$ A800 at c) low and d) high magnification; Starbon ${ }^{\circledR}$ A1000 e) low and f) high magnification; AMCS-NW g) low and h) high magnification and AMCS-W i) low and $\mathrm{j}$ ) high magnification. The white arrow in figure $1(\mathrm{~g})$ highlights a region of crystalline calcium. 


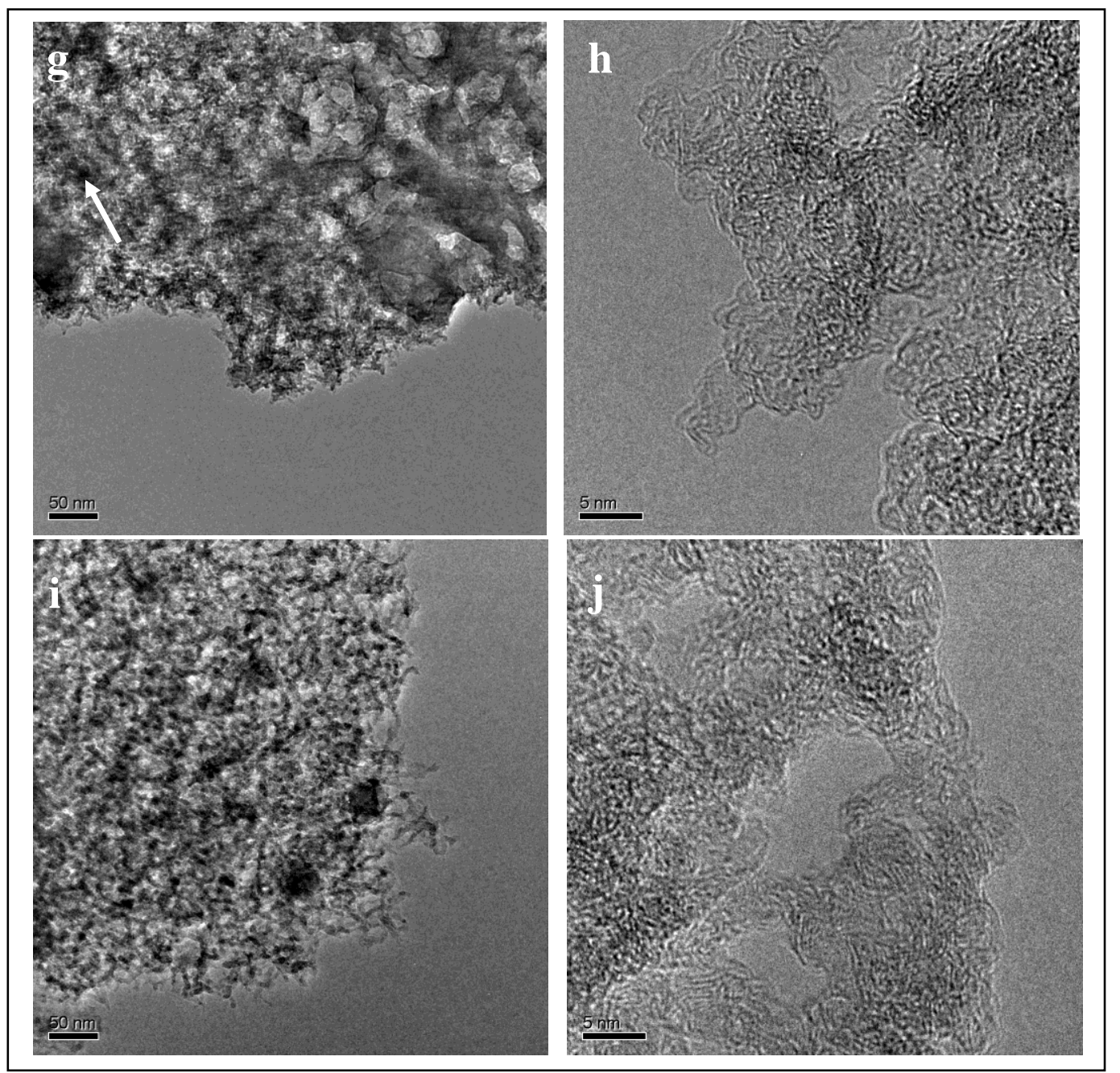

Figure 1: (continued)

It is clear that there are significant differences between the microstructures of the commercial PGC, alginic acid-derived Starbon ${ }^{\circledR}$ and AMCS materials. This makes the fact that they exhibit such similar properties in terms of chromatographic separation all the more interesting. In order to understand further the nature of the carbon environments present in these materials, core loss EELS data were collected at the carbon K-edge, which relates to transitions from the carbon $1 s$ core level to empty $p$-like final states above the Fermi level. Analysis of the carbon K-edge using the procedure described by Zhang et al. [13] is used to gain semi-quantitative information on the level of carbon $s p^{2}$ bonding from the relative intensity of the $\pi^{*}$ transition at $285 \mathrm{eV}$, and on the degree of fullerene character from the relative intensity of the residual peak at $287 / 287.5 \mathrm{eV}$. 


\subsection{2: Core loss EELS}

Representative EELS plots fitted at the carbon K-edge for Starbon ${ }^{\circledR}$ A800 and commercial PGC are shown in Figure 2 (a and b respectively). The percentage $s p^{2}$ content and percentage fullerene character data derived from these fitted plots using the procedure described by Zhang et $a l .{ }^{13}$ for all the samples are summarised in Table 2. The paper by Zhang et al describes the procedure for the extraction of the concept of "fullerene content". Essentially this is the relative intensity of the residual peak centred at ca. $287 \mathrm{eV}$ following a three Gaussian fit to the carbon K-edge. In practice, this peak is intensity is normalised to the total C K-edge intensity (integrated over a $20 \mathrm{eV}$ window) - the latter giving a measure of the total number of carbon atoms in the analysed volume. This relative residual peak intensity is then corrected by the baseline relative peak intensity in this region observed in a $100 \%$ planar $\mathrm{sp}^{2}$ reference material such as fully crystalline graphite (i.e. this is subtracted from the measured value). Finally the concept of "fullerene content" is achieved by normalising this ratio is to the corresponding ratio obtained from crystalline $\mathrm{C}_{60}$. In principle this normalised intensity ratio should reflect the degree of non-planar $\mathrm{sp}^{2}$-bonded carbon character relative to a perfect fullerene unit (some authors have termed this $\mathrm{sp}^{2+\mathrm{d}}$ character) - provided no other factors are contributing to this intensity such as carbon bonded to heteroatoms, the majority of which are expected to be driven off at the pyrolysis temperatures involved. 

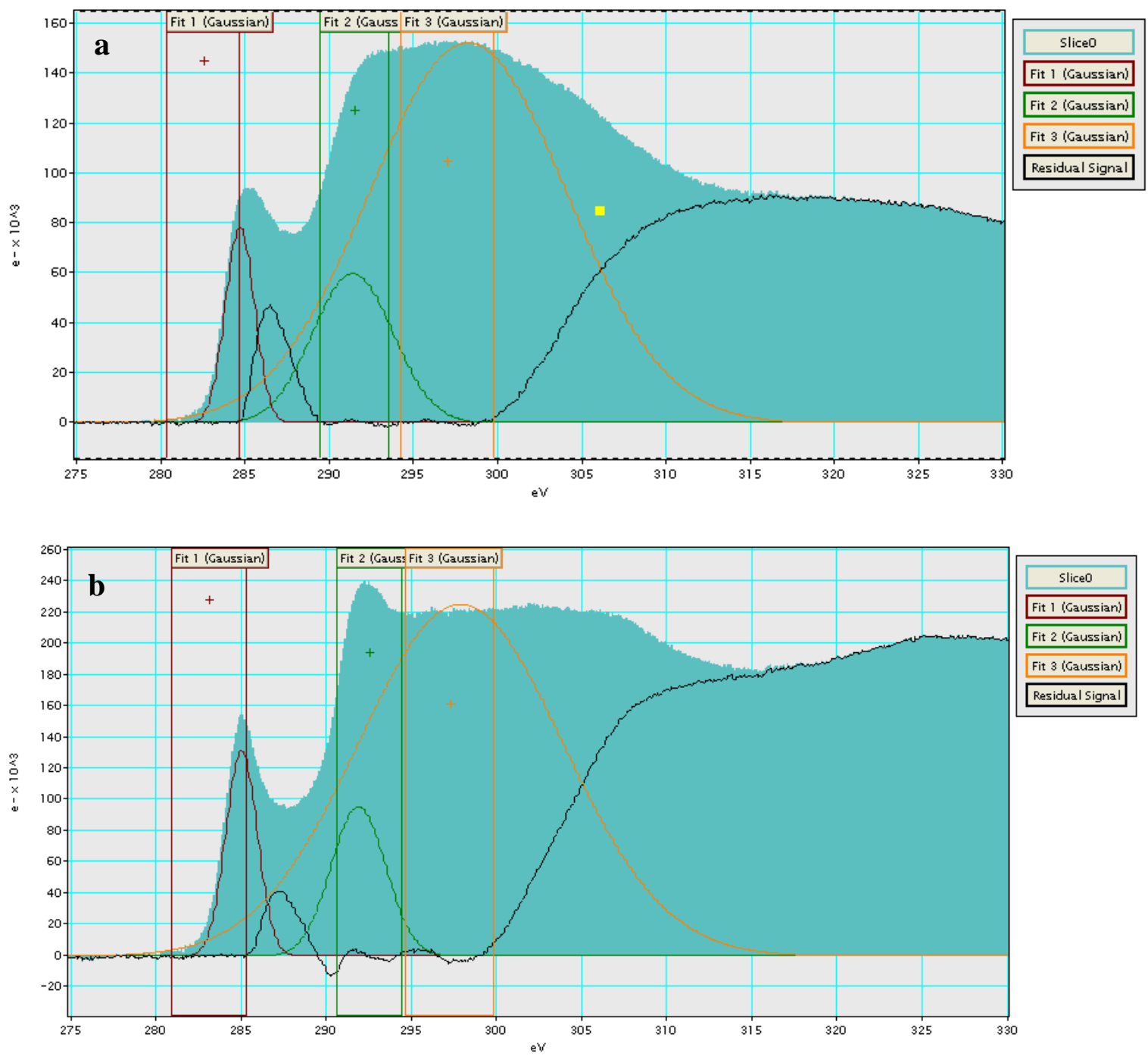

Figure 2: Fitted EELS plots at the carbon K-edge for a) Starbon ${ }^{\circledR}$ A800 and b) commercial PGC

The commercial PGC material exhibits an $s p^{2}$ content of greater than $90 \%$, which is expected, given the propensity of highly aligned graphitic layers in this material, and reveals a percentage fullerene content of around $30 \%$. This data appears to correlate with the evidence provided by the TEM images. This material shows similar though slightly lower values when compared with a phenolic resin-derived, non-graphitising carbon pyrolysed to $2500{ }^{\circ} \mathrm{C}$ investigated by Zhang et al. - that exhibited percentages of ca. $98 \%$ carbon $\mathrm{sp}^{2}$ content and $35 \%$ fullerene character respectively [13]. 


\begin{tabular}{ccc}
\hline Sample & $\begin{array}{c}\text { Percentage } s p^{2} \text { content } \\
/ \%\end{array}$ & $\begin{array}{c}\text { Percentage fullerene character } \\
\text { \% }\end{array}$ \\
\hline Commercial PGC & $90 \pm 4$ & $30 \pm 1$ \\
Starbon $^{\circledR}$ A800 & $72 \pm 2$ & $66 \pm 12$ \\
Starbon $^{\circledR}$ A1000 & $87 \pm 7$ & $55 \pm 16$ \\
AMCS-NW & $76 \pm 3$ & $53 \pm 5$ \\
AMCS-W & $82 \pm 4$ & $57 \pm 6$ \\
\hline
\end{tabular}

Table 2: Summary of core loss EELS data for all commercial PGC, AMCS and Starbon ${ }^{\circledR}$ samples. Errors represent the standard deviation of analysis from at least three different sample regions.

Starbon $^{\circledR}$ A800 shows a lower $s p^{2}$ content (of $72 \%$ ) than that observed in a corresponding phenolic resin-derived, non-graphitising carbon pyrolysed at the same temperature of $800{ }^{\circ} \mathrm{C}$ (ca. $84 \%$ ) [13]. Meanwhile Starbon ${ }^{\circledR}$ A1000 shows a carbon $s p^{2}$ content similar to that seen in a comparative, non-graphitising carbon pyrolysed at $1000{ }^{\circ} \mathrm{C}(87 \%$ and $92 \%$ respectively [13]). However, both Starbon ${ }^{\circledR}$ A800 and Starbon ${ }^{\circledR}$ A1000 samples show significantly higher levels of fullerene character compared to the standard phenolic resin-derived, nongraphitising carbons, with $66 \%$ and $55 \%$ compared to $47 \%$ and $26 \%$ for materials pyrolysed to $800{ }^{\circ} \mathrm{C}$ and $1000{ }^{\circ} \mathrm{C}$ respectively. The AMCS samples show similar values to the Starbon ${ }^{\circledR}$ materials, with fullerene character calculated to be in the range $50-60 \%$.

A recent study by Powles et al. [20] reported the use of molecular-dynamics simulations to describe how self-assembled $s p^{2}$-bonded carbon structures, like fullerenes, could arise from highly disordered amorphous precursors. They found that two key factors affected the organising capabilities of $s p^{2}$ carbon: (i) the geometry of the precursor system, and (ii) the density (thus void space) of the material. 
Initially considering this second factor, the materials in our work can be considered lowdensity bulk carbons and afford a greater void fraction than higher-density carbons e.g. annealed carbons. The simulated study by Powles et al. yielded a hypothetical highly curved $s p^{2}$ sheet model for low-density bulk carbons which was similar to that put forward by Harris et al. to describe the fullerene-like character of non-graphitizing microporous carbons, on the basis of TEM observations [21].

Meanwhile the geometry of the precursor system before pyrolysis is that of a linear polyuronide polymer consisting of two hexuronic acid residues: $\beta$-D-mannuronic acid and $\alpha$ L-guluronic acid. A recent study by Ross et al. reported the volatile products released by alginic acid and its calcium and sodium alginate salts during pyrolysis [22]. The study found that, for alginic acid and calcium alginate, the major volatile component released was furfural, along with smaller quantities of various cyclopentenones. Potentially, the C5 intermediates formed by modification of the hexuronic acid units during the generation of these volatiles may therefore act as the nucleation point and template for the growth of the highly fullerene character observed in our materials.

Further to this, the $s p^{2}$ content of the AMCS-W sample was found to be higher than that for Starbon $^{\circledR}$ A800 sample, with the AMCS-W sample having $s p^{2}$ levels that are on a par with those of a standard phenolic resin-derived, non-graphitising carbon. It is therefore possible that the calcium nanoparticles present throughout these materials may act as nuclei, catalysing the formation of $s p^{2}$-hybridised carbon used to grow the fullerene layers observed. The apparently contradictory observation, whereby the AMCS-W sample has a higher $s p^{2}$ content than the AMCS-NW sample, could suggest that the large calcium clusters observed in the 
TEM images of the unwashed material are less effective catalysts for fullerene formation than the more dispersed calcium observed in the washed AMCS sample.

\section{3: X-ray photoelectron spectroscopy (XPS)}

In order to determine the chemical nature of the calcium present in the alginic acid-derived Starbon ${ }^{\circledR}$ and AMCS materials, samples were analysed by X-ray photoelectron spectroscopy (XPS). Figure 3 (a) shows the Ca $2 p$ signal for the expanded precursor of the alginic acidderived Starbon ${ }^{\circledR}$ materials, which is composed of a a pair of spin-orbit split doublets, the $2 p_{3 / 2}$ and $2 p_{1 / 2}$ states, with a $2 p_{3 / 2}$ binding energy of $346.8 \mathrm{eV}$, indicative of predominantly CaOOR interactions. The expansion of alginic acid to a hydrogel is described by an ionotropic gelation process resulting from the binding of $\mathrm{Ca}^{2+}$ ions to areas of poly $\alpha$-L-guluronic acid present in the polysaccharide. This leads to the formation of a secondary structure described by the egg-box model [23] (Figure 4) and these data for the precursor samples are consistent with this model. We believe the source of the calcium in these materials to be primarily that incorporated during alginic acid extraction, during which water-soluble sodium alginate is separated from other polysaccharides present in the algae through gelation with calcium, followed by filtration of the mixture. Following pyrolysis to $800{ }^{\circ} \mathrm{C}$, features attributed to Caalginate (Ca-OOR) are lost and new states at 347.5 and $348.4 \mathrm{eV}$ emerge, attributed to $\mathrm{CaCO}_{3} / \mathrm{CaO}$ and $\mathrm{Ca}(\mathrm{OH})_{2}$ respectively. 

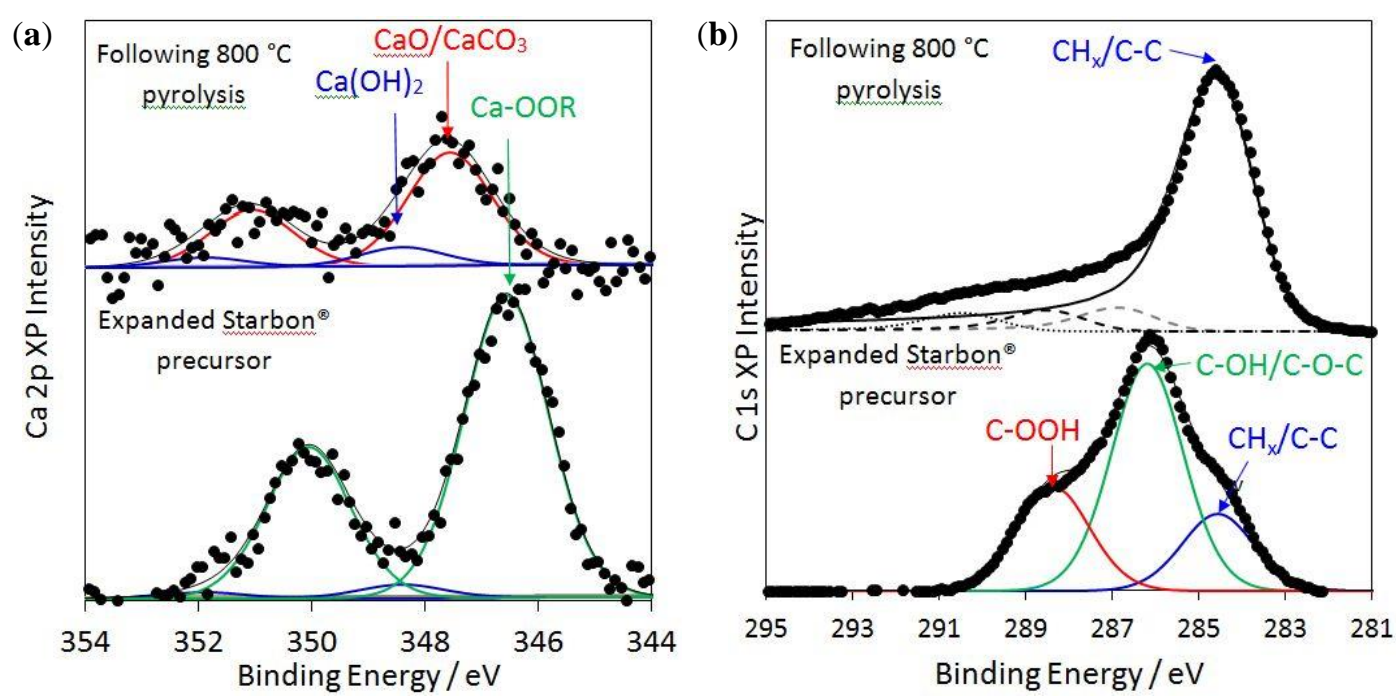

Figure 3: XPS analysis of expanded alginic acid-derived Starbon ${ }^{\circledR}$ precursor and pyrolysed to $800{ }^{\circ} \mathrm{C}$ (Starbon ${ }^{\circledast} \mathrm{A} 800$ ), in the a) $\mathrm{Ca} 2 p$ and b) $\mathrm{C} 1 s$ binding energy regions

The changes in the carbon environment following thermal treatment are shown in Figure 3 (b), which shows the parent alginate is comprised of three components at $284.6 \mathrm{eV}\left(\mathrm{CH}_{\mathrm{x}} / \mathrm{C}\right.$ C), $286.2 \mathrm{eV}(\mathrm{COC}, \mathrm{C}-\mathrm{OH}$,$) and 288.4 \mathrm{eV}(\mathrm{COOH})$ characteristic of the alginate backbone. The corresponding C $1 s$ photoelectron peak of the pyrolysed material shows that pyrolysis results in growth of the main peak at $284.5 \mathrm{eV}$ at the expense of the $\mathrm{COOH}$ and $\mathrm{COH} / \mathrm{C}-\mathrm{O}-\mathrm{C}$ components. The high degree of asymmetry in the new peak is attributed to a graphitic like species, having a characteristic $s p^{2}$ environment. Pyrolysis at $1000^{\circ} \mathrm{C}$ results in a sharpening of the C $1 s$ spectrum and a further decrease in oxygen content, as evidenced by the increased C:O ratio in Table 3. 


\begin{tabular}{|c|c|c|c|c|c|c|}
\hline \multirow[t]{2}{*}{ Sample } & \multicolumn{5}{|c|}{ Elemental atomic percentage / \% } & \multirow[t]{2}{*}{$\mathrm{C}: \mathrm{O}$ ratio } \\
\hline & $\mathbf{N a}$ & $\mathbf{N}$ & $\mathbf{O}$ & $\mathbf{C a}$ & $\mathbf{C}$ & \\
\hline Starbon ${ }^{(B)}$ precursor & 0.2 & 0.2 & 46.0 & 0.4 & 53.2 & 1.2 \\
\hline Starbon ${ }^{\circledR}$ A800 & 4.5 & - & 15.9 & 0.2 & 79.4 & 5.0 \\
\hline Starbon $^{\circledR}$ A1000 & - & 0.4 & 10.5 & 0.2 & 88.9 & 8.5 \\
\hline AMCS-NW precursor & - & - & 43.5 & 4.0 & 52.5 & 1.21 \\
\hline AMCS-NW & 0.3 & - & 36.4 & 9.2 & 54.1 & 1.48 \\
\hline AMCS-W precursor & - & - & 41.9 & 1.4 & 56.7 & 1.35 \\
\hline AMCS-W & - & - & 10.2 & 2.1 & 87.7 & 8.60 \\
\hline
\end{tabular}

Table 3: Surface compositions of Starbon ${ }^{\circledR}$ and AMCS samples

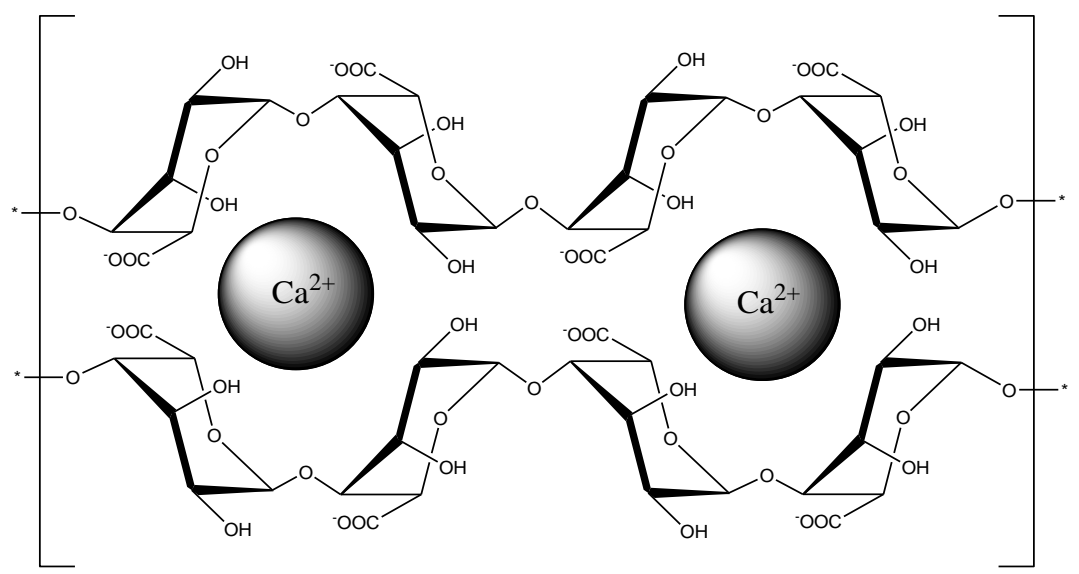

Figure 4: Representation of "egg-box" model binding of $\alpha$-L-guluronic acid blocks to calcium ions in alginic acid

Figure 5 (a and b) shows the $\mathrm{Ca} 2 p$ and $\mathrm{C} 1 s$ spectra, respectively, for the AMCS-NW precursor and AMCS-NW. In this instance, each of the Ca $2 p$ spin orbit split photoelectron lines is composed of a clear doublet, having $2 p_{3 / 2}$ components at 346.8 and 347.5 , attributed to Ca-OOR of alginate and $\mathrm{CaO} / \mathrm{CaCO}_{3}$ respectively. Following pyrolysis, the $\mathrm{CaO} / \mathrm{CaCO}_{3}$ state is retained; however the lower binding energy component is lost. There is an overall shift of the peak envelope to higher binding energy, due to the growth of a new set of doublets with Ca $2 p_{3 / 2}$ centred at $348.4 \mathrm{eV}$ which is attributed to $\mathrm{Ca}(\mathrm{OH})_{2}$. Following pyrolysis, growth of the $\mathrm{CH}_{\mathrm{x}} / \mathrm{C}-\mathrm{C}$ component in the $\mathrm{C} 1 s$ spectrum occurs at the expense of the $\mathrm{C}-\mathrm{OH}$ and $\mathrm{COOH}$ features, as the sample carbonizes. In addition, a new state emerges at $290.4 \mathrm{eV}$ which is 


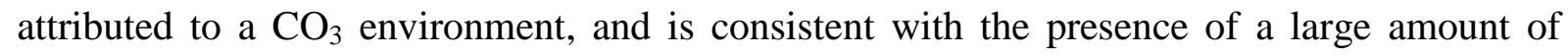
$\mathrm{CaCO}_{3}$ as indicated in the $\mathrm{Ca} 2 p$ spectrum. Powder X-ray diffraction was performed on the AMCS-NW sample in order to determine the $\mathrm{CaCO}_{3}$ phase type (Fig S1). Comparison of the sample diffraction pattern with those for $\mathrm{CaCO}_{3}$ recorded on the FIZ Karlsruhe ICSD suggests that the predominant polymorph is calcite, the pattern strongly correlated to that of Maslen et al. [24]. The peaks at $2 \theta=32.2^{\circ}, 37.4^{\circ}$ and $53.8^{\circ}$ could not be assigned although it is believed that they correspond to a minority crystal phase in this sample.
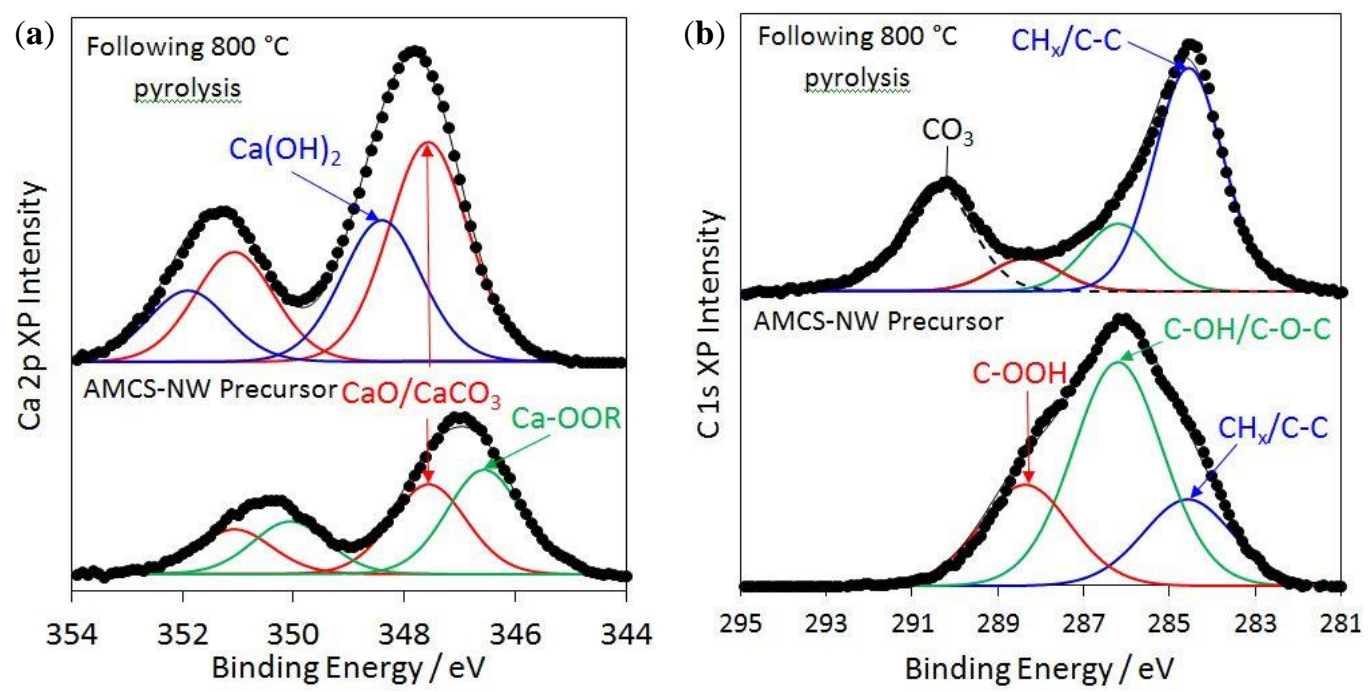

Figure 5: XPS analysis of AMCS-NW precursor and AMCS-NW, in the a) Ca $2 p$ and b) C $1 s$ binding energy regions

The effect of acid washing on pyrolysis was also investigated by XPS (Fig 6) which shows that, while a decrease in Ca content is observed (Table 3), there is no significant change in the surface carbon functionality (Fig 6b). Following pyrolysis at $800^{\circ} \mathrm{C}$, decomposition of the alginate is observed (as also in Figure 4), with the Ca $2 p$ exhibiting features for $\mathrm{Ca}(\mathrm{OH})_{2}$ and $\mathrm{CaCO}_{3}$. Inspection of the $\mathrm{C} 1 s$ now shows a single asymmetric peak for $\mathrm{CH}_{\mathrm{x}} / \mathrm{C}-\mathrm{C}$ similar to that observed for Starbon ${ }^{\circledR}$ A800 and Starbon ${ }^{\circledR}$ A1000. The loss of the peak at $290.4 \mathrm{eV}$, which was attributed to a carbonate-derived carbon, is consistent with a decrease in $\mathrm{CaCO}_{3}$ in the acid washed sample. The loss of $\mathrm{CO}_{3}$ is also manifest in the decreased oxygen content in the $800{ }^{\circ} \mathrm{C}$ pyrolysed sample (Table 3 ). 

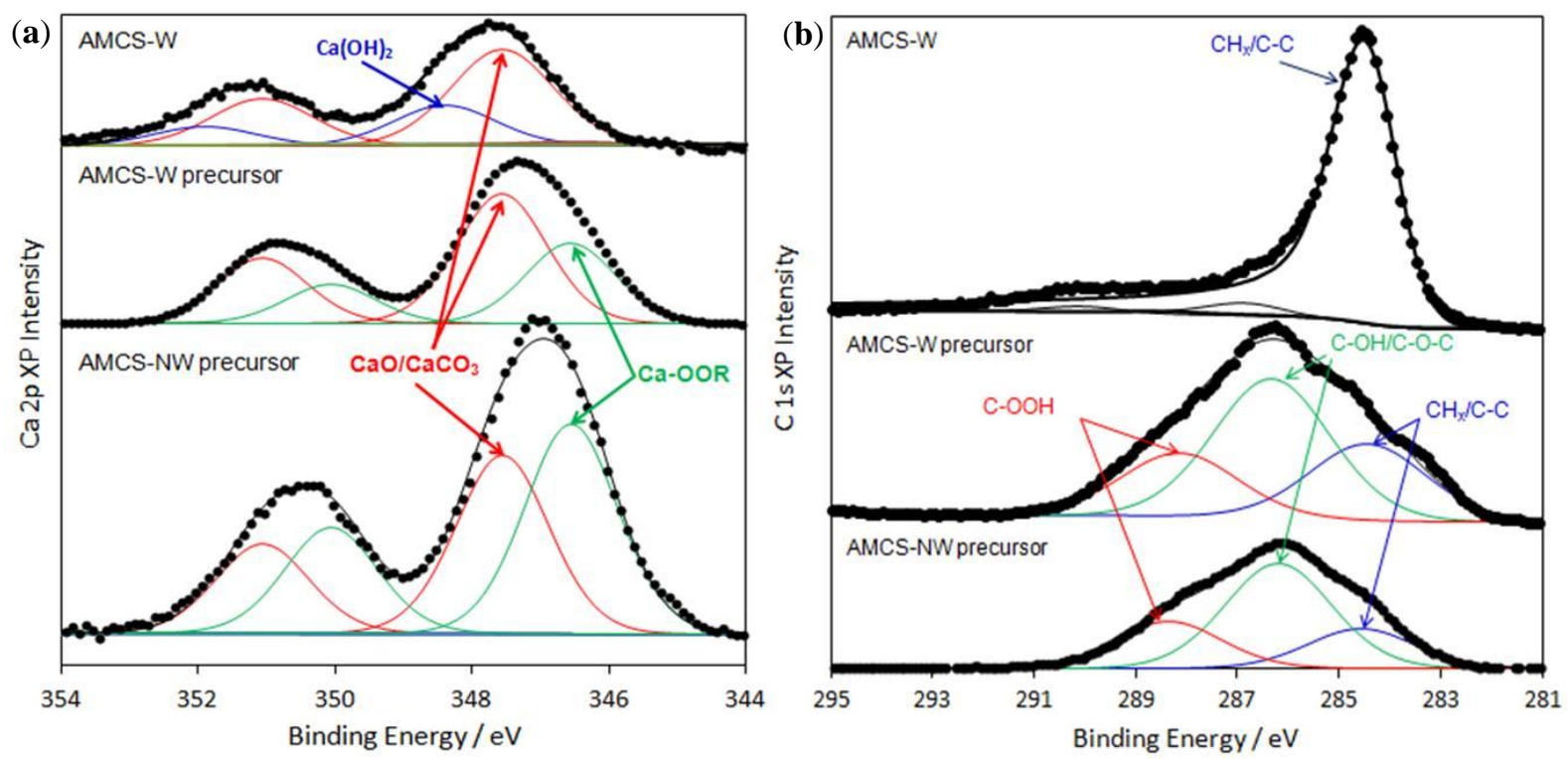

Figure 6: XPS analysis of AMCS-W precursor and AMCS-W, in the a) Ca $2 p$ and b) C $1 s$ binding energy regions

Analysis of the $s p^{2}: s p^{3}$ components of the Starbon ${ }^{\circledR}$ and AMCS samples was performed (based on fitted C $1 s$ peaks at 284.6 and $286.8 \mathrm{eV}$ respectively) using the procedure described by Díaz et al. [25] (Table 4). The results are broadly consistent with the deductions from HRTEM, that $\operatorname{Starbon}^{\circledR}$ A1000 contains a higher degree of $s p^{2}$ character than Starbon ${ }^{\circledR}$ A800, and is comparable with the AMCS-W sample. (N.B. The $286.8 \mathrm{eV}$ peak assigned to the $s p^{3}$ component overlaps with that for $\mathrm{C}-\mathrm{OH}$ so any surface oxidation may skew the results.)

\begin{tabular}{cc}
\hline Sample & ${ }^{a} \boldsymbol{s p ^ { 2 } : \boldsymbol { s p } ^ { 3 } \text { ratio }}$ \\
\hline Starbon $^{\circledR}$ A800 & 11.0 \\
Starbon $\left.^{(}\right)$A1000 & 22.7 \\
AMCS-NW & 13.0 \\
AMCS-W & 18.7 \\
\hline $\mathrm{a}=$ based on 284.6 and 286.8 eV components respectively &
\end{tabular}

Table 4: $s p^{2}: s p^{3}$ ratio for Starbon ${ }^{\circledR}$ and alginate AMCS samples 


\section{4: Potential mechanism for calcium catalysis}

Calcium catalysis of graphitisation processes is discussed widely in the literature, with a number of the articles describing processes whereby catalytic graphitisation of carbonising materials has occurred, albeit at high pressures of 3 kbar, where calcium was added. For example, the acceleration of graphite formation in polyvinyl chloride (PVC) cokes by calcium carbonate [26], hydroxide [27] and oxide [28], was described in the 1970s and products characterised by electron microscopy and X-ray diffraction (XRD). However, more recently, Tsubouchi et al. have described the catalysis of turbostratic graphite formation in several coal samples by calcium hydroxide under ambient pressures [29].

Two mechanisms of calcium catalysis have been postulated. The first, by Tsubouchi et al., describes a reaction by fine particles of $\mathrm{CaO}$ with amorphous carbon present in the forming chars, to produce $\mathrm{CO}$ and interstitial calcium carbide/oxide (i.e. $\mathrm{CaO}_{\alpha} \mathrm{C}_{\beta}$, where $\alpha$ and $\beta$ are fractional) intermediates [29]. The breakdown of this interstitial species then releases carbon in a graphitic form. This mechanism is represented as [29]:

$$
\mathrm{CaO}+\mathrm{C}_{\text {(amorphous) }} \longrightarrow \mathrm{CaO}_{\alpha} \mathrm{C}_{\beta} \longrightarrow \mathrm{CaO}_{\alpha} \mathrm{C}_{\gamma}+\mathrm{C}_{\mathrm{x} \text { (graphitic) }}
$$

The second mechanism, proposed by Hirano et al., describes an apparently cooperative effect between calcium carbonate and gaseous nitrogen in catalysing carbon graphitisation via a calcium cyanamide intermediate [26]:

$$
\begin{aligned}
\mathrm{CaCO}_{3} & \longrightarrow \mathrm{CaO}+\mathrm{CO}_{2} \\
\mathrm{CaO}+\mathrm{C}_{\text {(amorphous) }} & \longrightarrow \mathrm{Ca}+\mathrm{CO} \\
\mathrm{Ca}+2 \mathrm{C}_{\text {(amorphous) }} & \longrightarrow \mathrm{CaC}_{2} \\
\mathrm{CaC}_{2}+\mathrm{N}_{2} & \longrightarrow \mathrm{CaCN}_{2}+\mathrm{C}_{\text {(graphitic) }}
\end{aligned}
$$

In the case of the data presented in our paper, it is reasoned that the "graphitic" carbon described in the Hirano [26] and Tsubouchi [29] papers forms the fullerene-like structures 
observed in the alginic acid-derived Starbon ${ }^{\circledR}$ and AMCS materials. Since the carbon materials in this paper were prepared under a nitrogen atmosphere, it would be reasonable to assume that if calcium catalysis occurs, it may predominantly progress by the second mechanism. Indeed, the presence of high levels of calcium carbonate detected in our pyrolysed materials goes some way to supporting this view. Hirano et al. reported that graphitic particles were observed in pyrolysed PV-7 PVC by $1000{ }^{\circ} \mathrm{C}$, although the calcium carbonate infused into their starting material was in the form of "disks $8 \mathrm{~mm}$ in diameter and $2.5 \mathrm{~mm}$ thick" [26]. It is therefore conceivable that the nanoparticulate nature of the calcium speciesin the alginic acid-derived Starbon ${ }^{\circledR}$ and AMCS materials described here may enhance the catalytic effect still further. An earlier study by Hirano et al. reported a positive correlation between the extent of graphite formation and the distribution and concentration of calcium found in several specimens of PVC coke [30]. In the case of the unwashed AMCS material, a large proportion of calcium appears to be tied up in large, crystalline structures; the calcium is heterogeneously distributed though the sample, and the concentration of calcium available for catalysis at a particular position is thus lower than that of the unwashed AMCS material. This could explain why this unwashed AMCS material presented a reduced carbon $s p^{2}$ content than the washed AMCS sample.

\section{Discussion and conclusions}

Analysis of a commercial PGC material using TEM and core loss EELS spectroscopy indicates that it resembles a typical, high temperature-pyrolysed non-graphitising carbon derived from phenolic resin. However, analysis of two types of mesoporous carbon derived from the algal polysaccharide alginic acid (Starbon ${ }^{\circledR}$ and AMCS), shows a higher percentage fullerene character than the respective non-graphitising carbon. In addition, AMCS samples had a higher $s p 2$-content than the alginic acid-derived Starbon ${ }^{\circledR}$ material pyrolysed to $800{ }^{\circ} \mathrm{C}$. 
Based on these observations and the results of XPS analysis, a mechanism of calcium catalysis for the formation of fullerene-like carbon has been discussed.

The carbon microstructures of Starbon ${ }^{\circledR}$ and AMCS are considerably different to that of commercial PGC. Both Starbon ${ }^{\circledR}$ and AMCS have extensive curvature of graphitic layers due to high-fullerene character whilst the microstructure of PGC predominantly features extended planar graphitic stacks. The fact that the chromatographic properties of the two biomassderived carbons are similar to those of PGC suggests that, at the molecular level, the retentive properties of fullerene-like and turbostratic graphite, for an analyte, are equivalent. In addition, whilst the fullerene stacks of Starbon ${ }^{\circledR}$ and AMCS were limited to 2-3 layers, the graphitic stacks of PGC were 3-6 layers thick which suggests that the stack depth has minimal effect on the retention mechanism in PGC chromatography.

\section{Acknowledgements}

A. S. Marriott would like to thank the BBSRC for DTA doctorate funding. We also thank the EPSRC (Grant reference EP/K023853/1) for funding access to TEM facilities and expertise under the Leeds EPSRC Nanoscience Research Equipment Access Facility (LENNF) scheme.

\section{References}

[1] Gokulakrishnan N, Kania N, Léger B, Lancelot C, Grosso D, Monflier E et al., An ordered hydrophobic P6mm mesoporous carbon with graphitic pore walls and its application in aqueous catalysis. Carbon 2011; 49 (4): 1290-8

[2] Chai S- H, Howe JY, Wang X, Kidder M, Schwartz V, Golden ML et al., Graphitic mesoporous carbon as a support of promoted $\mathrm{Rh}$ catalysts for hydrogenation of carbon monoxide to ethanol. Carbon 2012; 50 (4): 1574-82 
[3] West C, Elfakir C, Lafosse M, Porous graphitic carbon: A versatile stationary phase for liquid chromatography. J. Chromatogr. A 2010; 1217 (19): 3201-16

[4] Saliger R, Fischer U, Herta C, Fricke J, High surface area carbon aerogels for supercapacitors. J. Non-Cryst. Solids 1998; 225: 81-5

[5] Fu R- W, Li Z- H, Liang Y- R, Li F, Xu F, Wu D- C, Hierarchical porous carbons: design, preparation, and performance in energy storage. New Carbon Mater. 2011; 26 (3): 171-9

[6] Gilbert MT, Knox JH, Kaur B, Porous Glassy Carbon, A New Columns Packing Material for Gas Chromatography and High-Performance Liquid Chromatography. Chromatographia $1982 ; 16(1): 138-46$

[7] Knox JH, Kaur B, Millward GR, Structure and performance of porous graphitic carbon in liquid chromatography. J. Chromatogr. A 1986; 352: 3-25

[8] P. Ross, R. E. Majors, LC-GC Eur. 2000; 13: 310-9

[9] Budarin V, Clark JH, Hardy JJE, Luque R, Milkowski K, Tavener SJ et al., Starbons: New Starch-Derived Mesoporous Carbonaceous Materials with Tunable Properties. Angew. Chem. 2006; 118 (23): 3866-70

[10] White RJ, Budarin VL, Clark JH, Tuneable Mesoporous Materials from $\alpha$-DPolysaccharides. ChemSusChem 2008; 1 (5): 408-11

[11] White RJ, Antonio C, Budarin VL, Bergström E, Thomas-Oates J, Clark JH, Polysaccharide-Derived Carbons for Polar Analyte Separations. Adv. Funct. Mater. 2010; 20 (11): 1834-41

[12] Antonio C, Pinheiro C, Chaves MM, Ricardo CP, Ortuño MF, Thomas-Oates J, Analysis of carbohydrates in Lupinus albus stems on imposition of water deficit, using porous graphitic 
carbon liquid chromatography-electrospray ionization mass spectrometry. J. Chromatogr. A 2008; 1187 (1-2): 111-8

[13] Zhang Z, Brydson R, Aslam Z, Reddy S, Brown A, Westwood A et al. Investigating the structure of non-graphitising carbons using electron energy loss spectroscopy in the transmission electron microscope. Carbon 2011; 49 (15): 5049-63.

[14] Parker HL, Hunt AJ, Budarin VL, Shuttleworth PS, Miller KL, Clark JH, The importance of being porous: polysaccharide-derived mesoporous materials for use in dye adsorption. RSC Advances. 2012; 2 (24): 8992-7

[15] Marriott AS, Hunt AJ, Bergström E, Wilson K, Budarin VL, Thomas-Oates J et al., Natural template approach to mesoporous carbon spheres for use as green chromatographic stationary phases. RSC Advances. 2013; Paper submitted

[16] Robitzer M, Di Renzo F, Quignard F, Natural materials with high surface area. Physisorption methods for the characterization of the texture and surface of polysaccharide aerogels. Micropor. Mesopor. Mat. 2011; 140 (1): 9-16

[17] Daniels H, Brown A, Scott A, Nichells T, Rand B, Brydson R, Experimental and theoretical evidence for the magic angle in transmission electron energy loss spectroscopy, Ultramicroscopy. 2003; 96 (3): 523-34

[18] NIST X-ray Photoelectron Spectroscopy Database, Version 4.1. Gaithersburg, 2012: National Institute of Standards and Technology. c2000 [updated 15th September 2012, cited June 2013]. Available from: http://srdata.nist.gov/xps/

[19] Harris PJF, Burian A, Duber S, High resolution electron microscopy of a microporous carbon. Philos. Mag. Lett. 2000; 80 (6): 381-6

[20] Powles RC, Marks NA, Lau DWM, Self-assembly of $s p^{2}$-bonded carbon nanostructures from amorphous precursors. Phys. Rev. B. 2009; 79 (7): 075430 
[21] Harris PJF, Tsang SC, High-resolution electron microscopy studies of non-graphitizing carbons. Phil. Mag. A. 1997; 76 (3): 667-77

[22] Ross AB, Hall C, Anastasakis A, Westwood A, Jones JM, Crewe RJ, Influence of cation on the pyrolysis and oxidation of alginates. J. Anal. Appl. Pyrol. 2011; 91 (2): 344-51

[23] Grant GT, Morris ER, Rees DA, Smith PJC, Thorn D, Biological interactions between polysaccharides and divalent cations: the egg-box model. FEBS Letters. 1973; 32 (1): 195-8

[24] Maslen EN, Streltsov VA, Streltsova NR, X-ray study of the electron density in calcite, $\mathrm{CaCO}_{3}$. Acta Crystallogr. B. 1993; 49 (4): 636-41

[25] Díaz J, Paolicelli G, Ferrer S, Comin F, Separation of the $s p^{3}$ and $s p^{2}$ components in the C1s photoemission spectra of amorphous carbon films. Phys. Rev. B; 54 (11): 8064-9

[26] Hirano S, Inagaki M, Saito H, Cooperative accelerating effect of calcium carbonate and gaseous nitrogen on graphitization of carbon. Carbon 1979; 17 (5): 395-8

[27] Noda T, Inagaki M, Hirano S, Saito H, Effect of coexisting minerals on graphitization of carbon under pressure. III. Accelerating effect of calcium hydroxide. Bull. Chem. Soc. Jpn. 1969; 42 (6): 1738-40

[28] Õya A, Õtani S, Effects of particle size of calcium and calcium compounds on catalytic graphitization of phenolic resin carbon. Carbon 1977; 17 (2): 125-9

[29] Tsubouchi N, Xu C, Ohtsuka Y, Carbon Crystallization during High-Temperature Pyrolysis of Coals and the Enhancement by Calcium. Energy and Fuels 2003; 17 (5): 1119-25

[30] Hirano S, Inagaki M, Saito H, Effect of Coexisting Minerals on the Graphitization of Carbon under Pressure. V. Distribution of Calcium and Graphitic Components in HeatTreated Carbon Specimens Bull. Chem. Soc. Jpn., 1970; 43 (8): 2624-5 
